\title{
MS36-04 | Pharmaceutical Solid Solutions from Non-Soluble Components
}

Lusi, Matteo (University of Limerick, Limerick)

Crystalline solid solutions of molecular species are a poorly understood class of material that allow the fine-tuning of structural and physicochemical properties, in continuum. Traditionally such phases are prepared according to simples guidelines as summarised by the Hume-Rotary and Kitaigorodskii set of rules. Similar size, isomorphicity of the parent component and molecular isostructurality are often deemed as a requirement for solubility in the solid state.

Recent works show the limits of the above-mentioned rules and defines alternative supramolecular and synthetic strategies for the development of solid solutions form "non-soluble" components. Incidentally, the new strategy enable crystalline solid solutions of pharmaceuticals for more effective and simpler therapies. 